\title{
一种可吸收止血纤丝的有效性和安全性评价
}

王玲爽 ${ }^{1}$, 童健星 ${ }^{1}$, 赵哲哲 ${ }^{1}$,杨晓春 ${ }^{2}$

1. 杭州协合医疗用品有限公司, 浙江 杭州 310018

2. 浙江大学药学院, 浙江 杭州 310058

[摘要]目的:评估可吸收止血纤丝的有效性和安全性。方法: 采用自主研发专 利技术制备得到一种可吸收止血纤丝, 与已上市产品速即纱进行比较, 通过大体观 察和傅里叶红外光谱法表征其物理形态及分子结构; 电位滴定法测定纤丝的羒基 含量, 酸碱度计测定酸碱度值, 铜乙二胺法测定相对分子质量; 倒置显微镜观察纤 丝与血液接触后的表现; 琼脂扩散法评价纤丝的细胞毒性。建立大鼠髂外动脉出 血模型和背部肌肉渗血模型, 通过止血时间和血液质量考察纤丝的止血有效性, 通 过观察拍照、免疫器官称重、血液学和凝血功能检测及组织病理学检查分析纤丝 的降解吸收情况和安全性。结果: 纤丝和速即纱具有相似的分子结构。与原料再 生纤维素相比, 纤丝和速即纱在 $1727 \mathrm{~cm}^{-1}$ 附近均出现了典型的羒基- $\mathrm{COOH}$ 伸缩振 动吸收峰。两种材料羒基含量约为 $20 \%$, 酸碱度值约为 3 , 与氧化前相比表现出明 显的酸性。纤丝经氧化后的相对分子质量为 $4466 \pm 79$, 与速即纱接近 $(P>0.05)$ 。纤 丝与速即纱接触血液后体积膨胀, 可吸收自身质量数十倍的血液。琼脂扩散实验 表明纤丝无细胞毒性。动物实验结果显示, 纤丝可在 $2 \mathrm{~min}$ 内完成止血, 止血时间和 吸收血液质量与速即纱差异无统计学意义 $($ 均 $P>0.05)$ 。纤丝植入肌肉渗血部位后 1 周即可降解, 植入纤丝对动物的外观体征、体重、摄食、胸腺、脾脏、淋巴结及 血液学、凝血功能等均无明显影响, 组织病理学检查未见明显异常。结论: 本研究 自主研发的可吸收止血纤丝具有较好的生物安全性和有效性。

[关键词] 纤维素; 止血材料; 纤丝; 可吸收; 安全性评价

[中图分类号 ] R318.08 [ 文献标志码 ] A

\section{Evaluation of self-prepared absorbable hemostatic cellulose fibrils}

WANG Lingshuang ${ }^{1}$, TONG Jianxing ${ }^{1}$, ZHAO Zhezhe ${ }^{1}$, YANG Xiaochun ${ }^{2}$ (1. Hangzhou Singclean Medical Products Co., Ltd, Hangzhou 310018, China; 2. College of Pharmaceutical Sciences, Zhejiang University, Hangzhou 310058, China)

Corresponding author: WANG Lingshuang, E-mail:wangls@hzxhe.com, https: //orcid.org/ 0000-0001-9360-5735

[ Abstract ] Objective: To evaluate the effectiveness and safety of self-prepared

收稿日期: 2021-03-12 接受日期:2021-07-27

作者简介: 王玲爽, 中级工程师, 主要从事医疗器械和材料研发工作;E-mail : wangls@hzxhe.com; https://orcid.org/00000001-9360-5735 
absorbable hemostatic fibrils. Methods: A kind of absorbable hemostatic fibrils were prepared by self-developed patent technique. The physical form and molecular structure of the fibrils and a marketed product Surgicel ${ }^{\circledR}$ were characterized by general observation and infrared spectroscopy; the carboxyl content, $\mathrm{pH}$ value and relative molecular mass of fibrils were determined by potentiometric titration method, $\mathrm{pH}$ meter and copper ethylenediamine method, respectively. The behavior of the fibrils and Surgicel ${ }^{\circledR}$ in contact with blood was observed by inverted microscope, the cytotoxicity was evaluated by agarose diffusion cell assay in vitro. The external iliac artery hemorrhage model and the back muscle infiltration model in rats were established. The hemostatic effectiveness of the fibrils was investigated by hemostasis time and blood weight, and the degradation and biosafety of fibrils were investigated by observation photography, immune organ weighing, hematology and coagulation index measuring, and histopathological examination. Results: The fibrils and Surgicel ${ }^{\circledR}$ had similar molecular structures. Compared with the raw material regenerated cellulose, the typical carboxyl stretching vibration absorption peak of $-\mathrm{COOH}$ appeared near $1727 \mathrm{~cm}^{-1}$ in both fibrils and Surgicel ${ }^{\circledR}$. The carboxyl content of the two materials was about $20 \%$, and the $\mathrm{pH}$ value was about 3 . The relative molecular mass of the fibers after oxidation was $4466 \pm 79$, which was close to that of Surgicel $^{\circledR}$ $(P>0.05)$. After contacting with blood, the volume of fibrils and Surgicel ${ }^{\circledR}$ expanded, and absorbed blood of dozens of times as their own weight. The results of agar diffusion test showed that the fibrils had no cytotoxicity. The results of animal experiments showed that the hemostasis completed within $2 \mathrm{~min}$, and there was no significant difference in blood weight and speed of hemostasis between two products (both $P>0.05$ ). The fibrils could be degraded 1 week after being implanted to the bleeding sites of the muscle. There were no pathological effects on the appearance, body weight, food intake, immunological tissue thymus, spleen, lymph nodes, hematology and coagulation indexes of the rats, and no obvious abnormality found in the histopathological examination. Conclusion: The prepared absorbable hemostatic fibrils have excellent biological safety and effectiveness.

[ Key words ] Cellulose; Hemostatic material; Fibril; Absorbable; Safety evaluation

[J Zhejiang Univ (Med Sci), 2021, 50(5): 633-641.]

可吸收止血材料在各种外科手术中已得到广 泛应用,理想的止血材料应具备止血迅速、无细胞 毒性、无免疫原性、不增加感染率、不影响组织愈 合等特点 ${ }^{[1]}$ 。可吸收止血材料的种类较多, 如纤 维素类、淀粉类、胶原蛋白及明胶类、壳聚糖类、纤 维蛋白原类等 ${ }^{[2]}$, 其中可吸收纤维素类止血材料 因其来源丰富、生物相容性较好, 且在体内可被快 速吸收, 在临床上占据一席之地。目前国内外已 上市的可吸收纤维素类止血材料主要有美国强生 公司的速即纱、北京泰科斯曼科技发展有限公司 的泰绫、贵州金玖生物技术有限公司的速绫等。
纤维素类材料主要以物理止血为主, 其接触血液 后能吸附血液中的水分, 血液黏稠度增大, 流速变 慢, 同时其自身膨胀形成胶状块封堵出血点,压迫 毛细血管末端达到止血目的。此外,因其表面上 羧基带有电荷, 可吸附和激活血小板, 触发内源性 凝血, 羧基还可能与血红素中的亚铁离子集合, 导 致非特异性血小板聚集,形成血凝块, 从而达到止 血目的 ${ }^{[3]}$ 。

纤维密度、织物组织和编织方式是影响材料 止血效率的重要因素。目前, 我国已上市的纤维 素止血材料以纱布状最为常见,但其单层结构限 
制其应用。进口产品如速即纱有纱布状、纤丝状 $\left(\right.$ Fibrillar $\left.^{\mathrm{TM}}\right)$ 等多种型号, 纤丝状止血材料因其具 有一定厚度,材料更蓬松柔软、纤维间空隙较多, 从而吸水性更强、血液黏附性好, 尤其适用于肿瘤 切除术、神经外科手术等。但由于其价格较昂贵, 因此市场尚需开发新的产品来丰富产品线, 降低 患者使用成本。

本研究采用二氧化氮氧化体系制备了一种具 有多层结构的可吸收止血纤丝( 以下简称纤丝), 并对其分子结构和氧化度进行表征, 观察其与血 液接触时的表现, 通过体外细胞实验和体内动物 实验评价其止血性能和生物安全性, 为其应用于 临床提供依据。

\section{1 材料与方法}

\section{1 材料与仪器}

小鼠成纤维细胞 L929购自中国科学院上海 细胞生物学研究所; 无特定病原体大鼠由北京科 奥协力饲料有限公司提供。MEM 培养基为美国 Thermo Fisher Scientific 公司产品; 胎牛血清为浙江 天杭生物科技股份有限公司产品; 青一链震素为北 京索莱宝科技有限公司产品; 含 $1 \%$ 肝素的猪新鲜 血液由浙江中医药大学安全评价中心提供; 速即 纱 $\left(\right.$ Surgicel $\left.{ }^{\circledR}\right)$ 为美国强生公司产品; 再生纤维素为 恒天海龙股份有限公司产品; 二氧化氮为中吴光 明化工研究院产品; 环已烷、乙醇、氢氧化钠等试 剂为浙江常青化工有限公司产品; 酮乙二胺溶液 为山东西亚化学工业有限公司产品; 丙三醇为国 药集团化学试剂有限公司产品; 纯化水为公司 自制。

傅里叶红外光谱仪 (Nicolet iS5)为美国 Thermo Fisher Scientific 公司产品; 电位滴定仪 (ET18)为美 国 Mettler Toledo 公司产品; 全自动血凝分析仪 (CA-550) 为日本 Sysmex 株式会社产品; 全自动血 液生化仪 (C311) 为瑞士 Roche Cobas 公司产品; 离 心机 (Eppendorf) 为德国Eppendorf公司产品; 电子 天平 (PL2001-L) 为美国 Mettler Toledo公司产品; 病理图像分析系统 (DM4000)为德国 Leica公司产 品; 酸碱度计 (FE-28)为美国 Mettler Toledo 公司产 品; 低温反应槽 (76-1A)为常州澳华仪器有限公司 产品; 毛细管粘度计(校准用内径 $0.57 \mathrm{~mm}$, 测定用 内径 $0.80 \mathrm{~mm}$ ) 为武汉市常青化工有限责任公司产 品; 倒置生物显微镜 (CKX53) 为日本 Olympus 公司
产品。

\section{2 纤丝的制备}

纤丝采用杭州协合医疗用品有限公司自主研 发的技术制备(专利授权号 201410848483.0 ), 具 体步骤如下: 首先将再生纤维素原料经开棉和弹 织制备具有多层结构的块状织物, 然后将织物置 于反应罐中, 预先在储罐中加人环己烷, 再将二氧 化氮通人储罐中调整至合适浓度后转移至反应罐 内进行氧化反应。反应结束后排出反应液, $95 \%$ 乙醇超声清洗4 6次,再加人无水乙醇脱水。随后 对其进行真空干燥 $12 \mathrm{~h}$ 以上,结束后裁切成适当大 小置于内包装纸中, 并放人铝箔袋进行封口, 得到 纤丝材料,将材料辐照灭菌后备用。

\section{3 纤丝理化特性表征}

1.3.1 大体观察及傅里叶红外光谱仪表征材料 物理形态、分子结构及氧化度 取适量纤丝、再生 纤维素和速即纱, 观察材料的物理形态, 然后利用 傅里叶红外光谱仪对三种材料的分子结构进行表 征, 观察和对比其吸收特征峰。材料的氧化程度 以羧基含量来表征,采用电位滴定法确定纤丝和 速即纱的羧基含量。

1.3.2 酸碱度计及铜乙二胺法测定材料酸碱度 和相对分子质量 纤丝、再生纤维素和速即纱三 种材料分别按每 $0.1 \mathrm{~g}$ 加水 $10 \mathrm{~mL}$, 室温放置 $1 \mathrm{~h}$ 后 过滤, 取滤液用酸碱度计检测酸碱度值。根据 GB/T 1548-2016《纸浆铜乙二胺 (CED) 溶液中特 性粘度值的测定》中酮乙二胺法检测三种材料 的相对分子质量,具体如下: 分别称取三种材料 各 $0.5 \mathrm{~g}$ 加人顶空瓶中, 先加人 $10 \mathrm{~mL}$ 水使其充分 解离后加人等量的铜乙二胺溶液, 加盖摇匀使其 充分溶解, 放人 $25^{\circ} \mathrm{C}$ 恒温水浴槽中。吸取 $3 \mathrm{~mL}$ 溶 液加人 $25^{\circ} \mathrm{C}$ 恒温的毛细管粘度计中, 平行测定两 次,记录流出时间,计算材料聚合度并换算相对 分子质量。

1.3. 3 倒置显微镜观察材料吸血量和膨胀程 度 将称量好的纤丝和速即纱分别置于培养正 中, 倒人小型猪新鲜血液, 等待材料吸收血液至饱 和,用镊子夹起材料称重,计算吸血量和吸血倍数 (吸血量/材料吸血前质量), 采用倒置显微镜观察 并测量材料单根纤维素短纤维吸血前后的直径。

\section{4 琼脂扩散试验测定纤丝细胞毒性}

根据GB/T 16886. 5-2017《医疗器械生物学 评价第 5 部分: 体外细胞毒性试验》 ${ }^{[4]}$ 中的方法, 
采用小鼠成纤维细胞L929来评价纤丝的细胞毒 性。选择生长旺盛的五代细胞, 消化后配置成 $1 \times 10^{5} / \mathrm{mL}$ 的细胞悬液。取六孔板, 每孔加入 $3 \mathrm{~mL}$ 细胞悬液, 于 $5 \%$ 二氧化碳、 $37{ }^{\circ} \mathrm{C}$ 下培养 $24 \mathrm{~h}$ 。弃 去培养液, 加人 $3 \mathrm{~mL}$ 琼脂培养基, 待凝固后, 加人 $3 \mathrm{~mL}$ 中性红染料, 培养 $30 \mathrm{~min}$, 吸除多余染料。将 材料制备成直径约 $5 \mathrm{~mm}$ 的圆片, 小心放置在琼脂 中心, 于 $5 \%$ 二氧化碳、 $37{ }^{\circ} \mathrm{C}$ 下培养 $24 \mathrm{~h}$, 同法制备 阴性对照 (无钙离子和镁离子的磷酸盐缓冲液) 和 阳性对照 $(5 \mathrm{~g} / \mathrm{L}$ 苯酚溶液)。继续培养 $24 \mathrm{~h}$ 后, 用 倒置显微镜观察试验材料正下方和周围细胞的生 长状况及染色情况, 根据细胞毒性反应级别进行

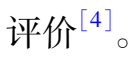

1.5 动物实验评价材料的止血效果、吸收情况 及安全性

动物实验委托浙江大学药物安全评价研究中 心进行, 实验过程均遵循单位和国家有关实验动 物管理和使用的规定。实验选用无特定病原体健 康成年大鼠120只, 体重200 240 g, 随机分为无菌 纱布组、纤丝组和速即纱组, 每组 40 只。选择髂外 动脉出血模型评价纤丝材料的止血效果, 具体步 骤如下: 动物麻醉后, 开腹, 用无菌针头刺破右侧 髂外动脉出血后, 分别用无菌纱布、纤丝和速即纱 贴于出血位置, 吸取流出的血液, 直到不能吸收血. 液为止。记录各组总出血量和止血时间。

通过大鼠背部肌肉渗血模型来评价材料的吸 收性能和安全性, 具体步骤如下: 在各组大鼠左侧 背部肌肉切开约 $1 \mathrm{~cm}$ 伤口, 造成渗血, 无菌纱布组 直接关腹缝合, 纤丝组和速即纱组分别覆盖相应 止血材料(使用剂量同无菌纱布组)后关腹缝合, 术后均给予抗菌药物预防感染。材料吸收情况的 评价方法: 大鼠术后第 $1 、 2 、 3 、 4$ 周分别麻醉取血, 处死后行解剖、拍照、肉眼观察材料降解及脏器异 常情况。材料的安全性评价方法: 大鼠术后连续 四周每周观察活动能力等健康状况, 并测体重和 摄食量。各组动物每周麻醉后腹主动脉取血 $3 \mathrm{~mL}$, 检测血液学一般指标和凝血功能; 各组每周 剖杀 10 只, 取大鼠肠系膜淋巴结、脾脏、胸腺称重, 计算脾脏系数和胸腺系数, 脾脏或胸腺指数 $=($ 脾 脏或胸腺质量/大鼠体重 $) \times 10$, 正常值范围参照文 献 $[5]$ 。然后用 $10 \%$ 甲醛溶液固定, 采用苏木精一 伊红染色法进行染色, 随后进行切片观察和组织 病理学检查。

\section{6 统计学方法}

采用SPSS 25.0 软件进行统计分析。计量数 据以均数 \pm 标准差 $(\bar{x} \pm s)$ 表示, 组间比较采用 Dunnett's 双侧 $t$ 检验, $P<0.05$ 为差异有统计学 意义。

\section{2 结 果}

\section{1 纤丝物理形态、分子结构及羧基含量}

纤丝为白色的块状固体, 具有一定厚度, 由若 干层再生纤维素短纤维层交错复合而成,材料柔 软蓬松(图 1), 吸水性强、使用量可控, 操作方便。

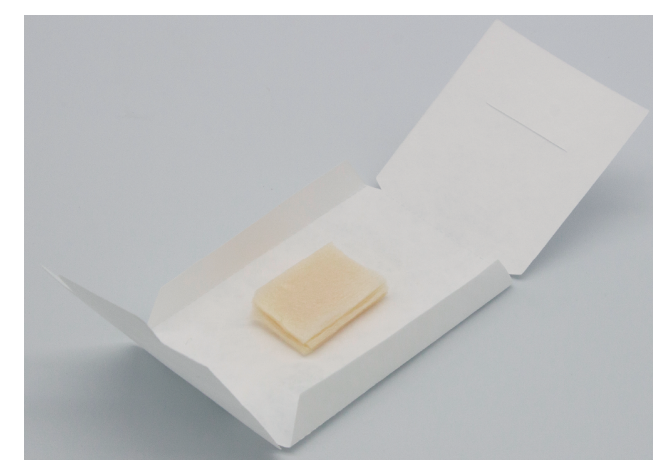

图 1 纤丝的外观

Figure 1 Appearance of fibrils

与再生纤维素相比,纤丝的红外图谱除在 $1727 \mathrm{~cm}^{-1}$ 处 (羧基 $-\mathrm{COOH}$ 的伸缩振动吸收峰)有 一明显的特征峰以外,其余基本相同。可见再生 纤维素经氧化后分子结构发生变化, 分子中产生 羧基。纤丝与速即纱的红外图谱相似,其中 3000 $3600 \mathrm{~cm}^{-1}$ 处出现了一个大宽峰, 为羟基 $\mathrm{O}-\mathrm{H}$ 伸缩 振动吸收峰, $(1155 \pm 10) 、(1017 \pm 10) \mathrm{cm}^{-1}$ 处出现 的吸收峰为 $\mathrm{C}-\mathrm{O}-\mathrm{C}$ 的伸缩振动吸收峰 ${ }^{[6]}$, 见 图 2 。经检测, 速即纱和纤丝的羒基含量分别为 $(20.2 \pm 1.2) \%$ 和 $(21.2 \pm 0.6) \%$, 差异无统计学意 义 $(P>0.05)$ 。

\section{2 纤丝酸碱度及相对分子质量}

与再生纤维素比较, 速即纱和纤丝的酸碱度 值和相对分子质量均减小 (均 $P<0.05)$, 而速即纱 与纤丝差异无统计学意义 $(P>0.05)$, 见表 1 。结果 提示, 再生纤维素经氧化后纤维素分子键发生断 裂, 导致相对分子质量显著降低, 产生的羧基导致 材料酸碱度值减小。

\section{3 纤丝吸血倍数及膨胀程度}

速即纱的吸血倍数为 24.5 倍和 28.3 倍, 纤丝 


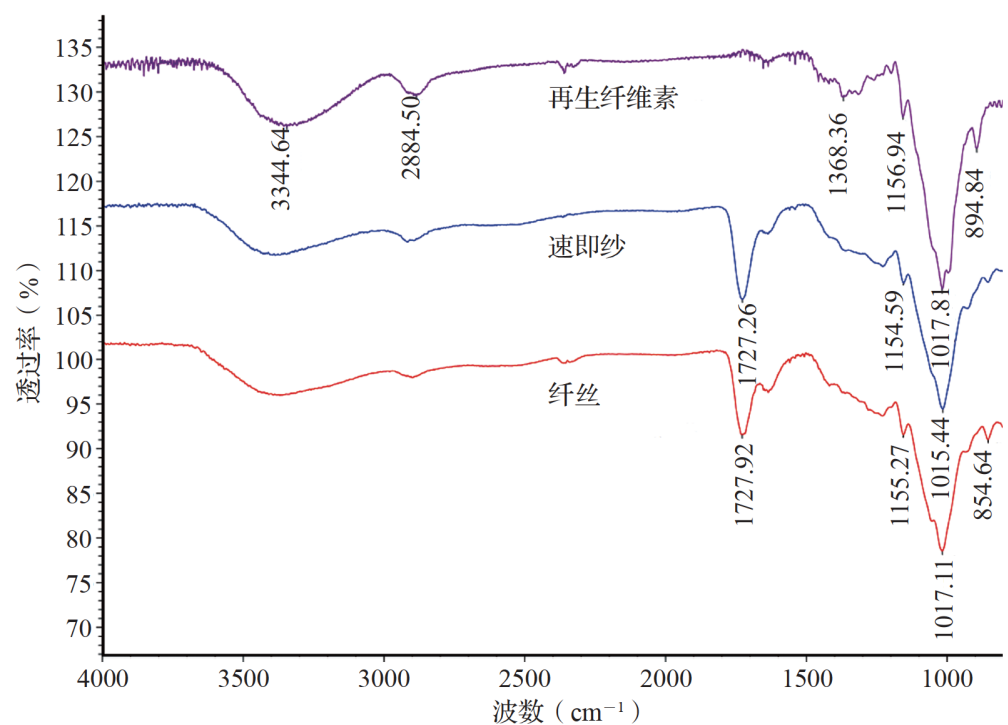

图 2 再生纤维素、速即纱和纤丝的红外光谱图

Figure 2 Infrared spectra of regenerated cellulose, Surgicel ${ }^{\circledR}$ and fibrils
缓冲液正下方及其周围无反应 区域,细胞毒性反应分级为 0 ; 阳性对照苯酚溶液反应区域超 出试样尺寸 $1.0 \mathrm{~cm}$ 以上, 毒性反 应分级为 4 ,为重度细胞毒性; 纤丝周围及其下方也无反应区 域,毒性反应分级为 0 。结果提 示, 纤丝无细胞毒性。

\section{5 纤丝止血效果}

无菌纱布组、速即纱组和 纤丝组止血时间分别为 $(90 \pm$ $18) 、(84 \pm 14)$ 和 $(75 \pm 14) \mathrm{s}$, 速 即纱组和纤丝组止血时间较无 菌纱布组均缩短 $($ 均 $P<0.05)$; 无菌纱布组、速即纱组和纤丝 组出血量分别为 $(3.6 \pm 1.0)$ 、
表 1 再生纤维素、速即纱和纤丝的酸碱度及相对分子质量

Table 1 The $\mathrm{pH}$ value and molecular weight of regenerated cellulose, Surgicel $^{\circledR}$ and fibrils

$(\bar{x} \pm s)$

\begin{tabular}{llcc}
\hline \multicolumn{1}{c}{ 材 料 } & $n$ & 酸碱度 & 相对分子质量 \\
\hline 再生纤维素 & 3 & $5.35 \pm 0.11$ & $34699 \pm 188$ \\
速即纱 & 3 & $2.94 \pm 0.01^{*}$ & $4203 \pm 142^{*}$ \\
纤丝 & 3 & $2.99 \pm 0.03^{*}$ & $4466 \pm 79^{*}$ \\
\hline
\end{tabular}

与再生纤维素比较, ${ }^{*} P<0.05$.

的吸血倍数为 24.0 倍和 30.5 倍, 两者相近 (表 2 )。 倒置显微镜下观察, 速即纱单根纤维素短纤维膨 胀后由近 $100 \mu \mathrm{m}$ 增加到 $200 \mu \mathrm{m}$ 以上, 纤丝单根纤 维素短纤维膨胀后由70 90 $\mu \mathrm{m}$ 增加到近200 $\mu \mathrm{m}$, 两种材料单根纤维膨胀倍数均在 2 倍以上 (图 3 )。 结果提示, 纤丝与速即纱在接触血液后表现相似, 均可吸收自身质量数十倍的血液, 体积明显 膨胀。

\section{4 纤丝细胞毒性}

通过倒置显微镜观察发现, 阴性对照磷酸盐

表 2 速即纱和纤丝的吸血量及吸血倍数

Table 2 Blood absorption capacity of Surgicel ${ }^{\circledR}$ and fibrils

\begin{tabular}{lcccc}
\hline 材 料 $\begin{array}{c}\text { 吸血前质量 } \\
(\mathrm{g})\end{array}$ & $\begin{array}{c}\text { 吸血后质量 } \\
(\mathrm{g})\end{array}$ & $\begin{array}{c}\text { 吸血量 } \\
(\mathrm{g})\end{array}$ & 吸血倍数 \\
\hline 纤丝 & 0.11 & 2.75 & 2.64 & 24.0 \\
& 0.14 & 4.41 & 4.27 & 30.5 \\
速即纱 & 0.10 & 2.55 & 2.45 & 24.5 \\
& 0.10 & 2.93 & 2.83 & 28.3 \\
\hline
\end{tabular}

$(2.6 \pm 0.9)$ 和 $(2.5 \pm 1.0) \mathrm{g}$, 速即纱组和纤丝组出 血量较无菌纱布组均减小 $($ 均 $P<0.05)$ 。结果提 示, 纤丝和速即纱能够快速止血, 减少伤口出 血量。

\section{6 纤丝体内降解吸收情况}

大鼠术后第 1 周, 肌肉渗血部位的速即纱和 纤丝基本被降解吸收,组织表面未见明显残留,各 脏器外观未见明显异常; 术后第2、3、4周,植人部 位各脏器的外观未见明显异常(图4), 表明材料的 植人不会对组织器官造成较大影响。

纤丝

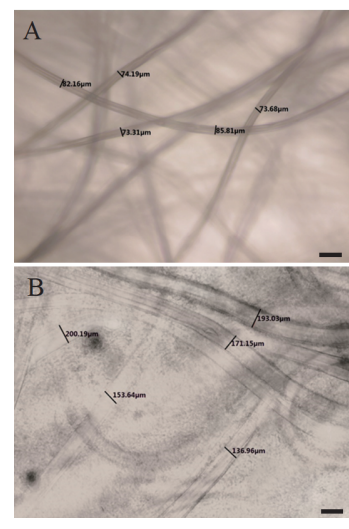

纤丝单根纤维素短纤维吸收血液前直径约70 90 $\mu \mathrm{m}(\mathrm{A})$, 吸收血液后膨胀至近 $200 \mu \mathrm{m}(\mathrm{B})$; 速即纱单根纤维素短纤维 吸收血液前直径约 $100 \mu \mathrm{m}(\mathrm{C})$, 吸收血液后膨胀至 $200 \mu \mathrm{m}$ 以上(D). 标尺 $=200 \mu \mathrm{m}$.

图 3 速即纱及纤丝在显微镜下吸血前后纤维直径 对比

Figure 3 Diameters of Surgicel ${ }^{\circledR}$ and fibrils before and after blood suction under the microscope 

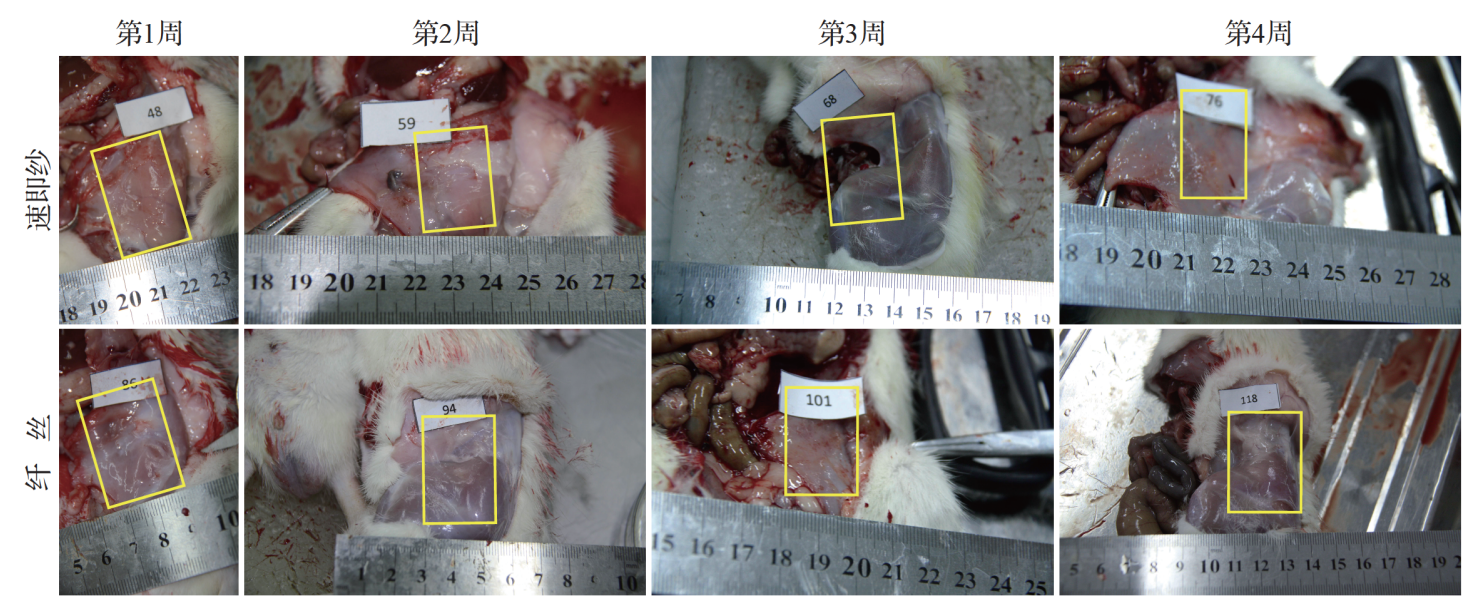

术后第1周,速即纱和纤丝在肌肉渗血部位基本被降解吸收,组织表面未见明显残留,各脏器外观未见明显异常; 术后第2、3、4 周,植人部位各脏器的外观未见明显异常. 黄色框区域为材料植人部位.

图 4 大鼠术后肌肉渗血部位材料降解情况

Figure 4 Degradation of materials in the muscle bleeding sites of rats

\section{7 纤丝的体内安全性}

整个试验过程中大鼠状态良好,摄食饮水正 常, 体重增长正常, 脾脏和胸腺的质量均在正常范 围(图 5、表 3), 各组术后胸腺、脾脏和肠系膜淋巴 结组织病理学检查均正常 (图6), 提示植人纤丝未 对组织造成不利影响。血液学和凝血功能检测结 果各组均在正常范围(表4), 各组差异无统计学意 义, 提示植人纤丝安全性较好。

\section{3 讨 论}

随着材料学和化学合成技术的进步,医用可 吸收止血材料的质量越来越好, 性能越来越优 异。目前,临床上广泛应用的纤维素止血产品速 即纱是由美国强生公司生产的一种氧化再生纤维 素速即纱,属于羧基纤维素类止血材料。其被血. 液完全浸透后会膨胀成浅棕色或黑色的胶状块, 该胶状块有助于形成凝块, 从而压迫血管达到止
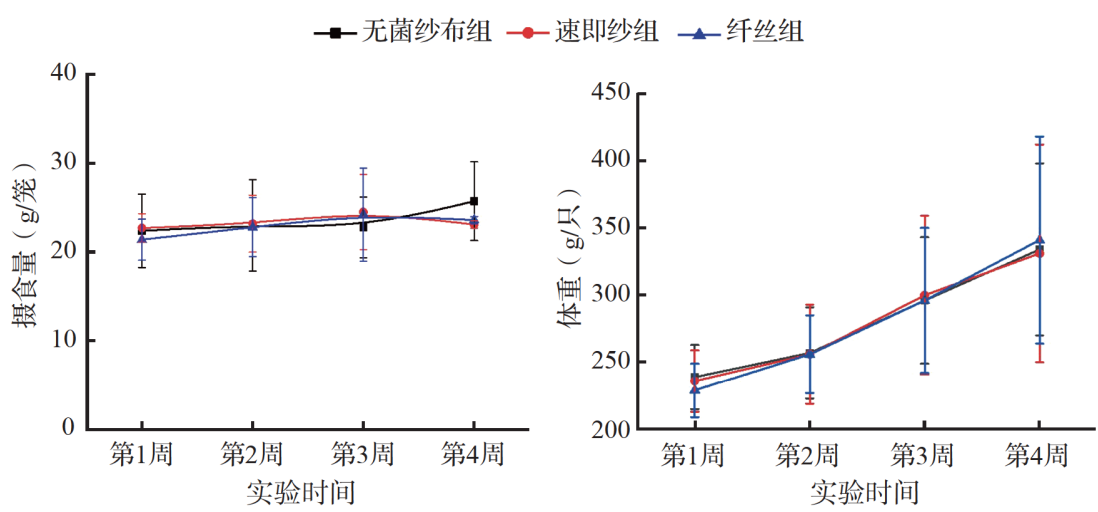

图 5 各组摄食量和体重增长情况

Figure 5 Food intake and weight gain of rats in each group
血目的。临床上速即纱应用于多个科室的手术止 血, 术中操作简便、易贴附于出血创面、可防组织 粘连, 且止血快、无毒副作用。速即纱贴敷 $10 \mathrm{~s}$ 后 即于接触处快速溶解, 变为胶体, 此时黏附力变 强, 1 3 $\mathrm{min}$ 后创面渗血逐渐减少至停止, 在人体 内最终分解成单糖而被吸收,其生物相容性与纤 维素的羧基含量有关 ${ }^{[7]}$ 。

氧化再生纤维素止血材料在形态上有纱布状 和纤丝状, 其中纤丝状氧化再生纤维素相比普通 纱布状氧化再生纤维素在止血方面优势明显。彭 承宏 ${ }^{[8]}$ 将氧化再生纤维素用于肝切除手术, 提出 就组织附着性和顺应性来看, 纤丝状速即纱优于 纱布状速即纱。纤丝状速即纱以其良好的附着性 和可塑性得到临床广泛应用。黄伟等 ${ }^{[9]}$ 比较了氧 化再生纤维素纱布与纤丝的吸水率、凝胶化速率、 溶解速率、降解速率、抗老化性能和止血效果, 结 果显示, 在相同氧化度条件下, 纤丝饱和吸水率比 纱布高 $50 \%$ 130\%, 纤丝在 $5 \mathrm{~s}$ 内的吸水率达 $500 \%$, 而 纱布只有 $250 \%$ 左右, 纤丝 松散网状多孔结构有利于 提高吸水率和吸水速率, 加 快凝胶化速率和溶解速率, 止血效果更好。本研究制 备的材料是一种止血纤丝, 外观上看, 为具有一定厚度 的多层织物, 蓬松柔软, 这 种结构使其在术中使用更 
表 3 各组术后第1周和第4周胸腺、脾脏称重结果比较

Table 3 The weight of thymus and spleen at the first and the fourth weeks after operation in each group

$(\bar{x} \pm s)$

\begin{tabular}{rrrcccc}
\hline 组 & 别 & $n$ & 胸腺 $(\mathrm{g})$ & 脾脏 $(\mathrm{g})$ & 胸腺系数 & 脾脏系数 \\
\hline 无菌纱布组 & 第1周 & 39 & $0.576 \pm 0.159$ & $0.912 \pm 0.157$ & $0.220 \pm 0.040$ & $0.354 \pm 0.052$ \\
& 第4周 & 10 & $0.504 \pm 0.060$ & $0.728 \pm 0.190$ & $0.154 \pm 0.029$ & $0.216 \pm 0.032$ \\
\multirow{2}{*}{ 速即纱组 第1周 } & 39 & $0.524 \pm 0.149$ & $0.807 \pm 0.272$ & $0.206 \pm 0.054$ & $0.314 \pm 0.091$ \\
& 第4周 & 8 & $0.480 \pm 0.091$ & $0.728 \pm 0.145$ & $0.147 \pm 0.034$ & $0.226 \pm 0.066$ \\
\multirow{2}{*}{ 纤丝组 } & 第1周 & 30 & $0.416 \pm 0.147$ & $0.757 \pm 0.185$ & $0.168 \pm 0.052$ & $0.300 \pm 0.048$ \\
& 第4周 & 7 & $0.411 \pm 0.066^{*}$ & $0.835 \pm 0.178$ & $0.120 \pm 0.013^{*}$ & $0.254 \pm 0.127$ \\
\hline
\end{tabular}

与无菌纱布组比较, ${ }^{*} P<0.05$.

加便捷。医生可根据创面大小和形态取用适当大 小和层数的纤丝覆盖创面, 材料内部短纤维交错 堆叠的蓬松结构使其具备充足的空隙, 吸水性更 强, 血液黏附性好, 更易贴合于伤口表面, 为实现 快速止血奠定了基础。通过红外分析, 本研究中 纤丝材料的分子结构与速即纱类似, 与未氧化的再 生纤维素相比,具有明显的氧化特征峰。

纤维素因其不溶于水,且人体内无分解酶而 不能在人体内降解, 但经过氧化后的纤维素可以 在生物体内降解 ${ }^{[10]}$, 其中羧基含量可反映再生纤 维素的氧化程度。Ashton 等 ${ }^{[11]}$ 研究表明, 当氧化 再生纤维素的羧基含量达到 3\%时就具有生物可 吸收性, 且止血性能和吸收性能随着羧基含量的 增加而增加, 随聚合度的降低而增加, 并且随着降
胸 腺

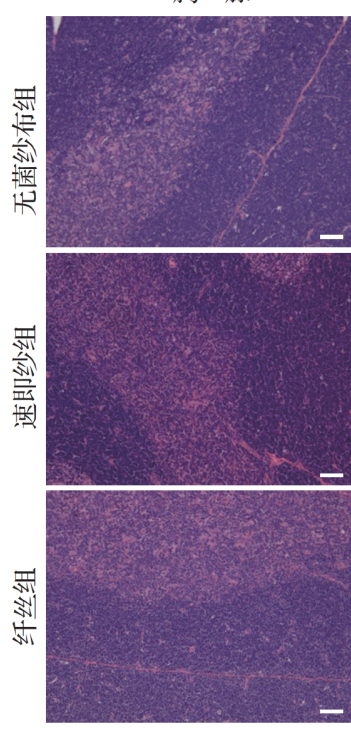

各组各脏器组织结构均正常, 未见明显差异. 标尺 $=50 \mu \mathrm{m}$.
脾 脏

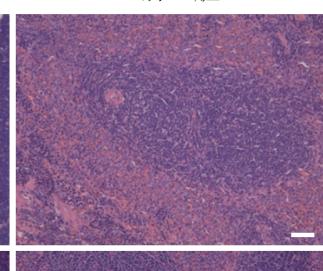

图 6 术后第4周各组胸腺、脾脏和肠系膜淋巴结组织病理学表现

Figure 6 Histopathological pictures of thymus, spleen and mesenteric lymph nodes in each group at the 4 th week after operation
解时间的延长, 氧化再生纤维素的降解程度也相 应增大。但如果氧化程度过高,则可能会因为纤维 素的降解速度过快导致局部组织渗透压过高,引发 炎症。因此,可吸收纤维素类材料的羧基含量一般 控制在 $16 \%$ 24\% ${ }^{[12]}$ 。经测定, 本研究制备的纤丝 和速即纱的羧基含量均在 $20 \%$ 左右,相对分子质 量约为 4000 , 表明其氧化程度接近, 氧化度适中。 氧化后的纤丝材料酸碱度值约为 3 , 具有广谱杀菌 的作用, 能够在一定程度上预防术后感染 ${ }^{[13]}$ 。实 验证明,纤丝和速即纱与血液接触后单根纤维膨 胀明显, 吸血倍数可达20 30 倍, 表现出了优异的 吸血性能。

本文资料显示, 纤丝无细胞毒性, 细胞相容性 好。本研究从止血性能和生物降解性能两方面对
肠系膜淋巴结

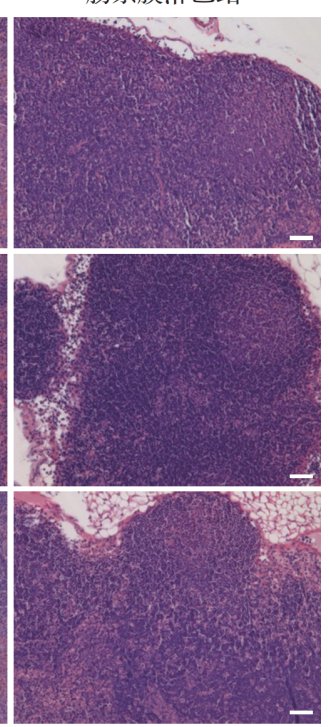

材料进行了动物体内评价。 与普通纱布相比,纤丝的止血 速度较快,出血量较无菌纱布 组更少,表明纤丝在实现快速 止血的同时可在一定程度上 抑制出血。研究通过构建大 鼠背部肌肉渗血模型,进一步 探讨了纤丝的降解性能以及 纤丝对组织的影响。大鼠术 后 1 4 周的解剖结果显示, 纤 丝在植人 1 周后大鼠背部已 无明显可见的肉眼残留,表明 纤丝在大鼠体内基本可在一 周内完成降解,与文献报道一 致 ${ }^{[14-15]}$ 。此外,本文资料还显 示,纤丝植人对大鼠摄食和体 重增长无明显影响,也未对大 鼠的血液和组织器官造成不 
表 4 各组术后第1周和第4周血液学和凝血功能检测结果比较

Table 4 Hematology and coagulation index at the 1st week and 4th weeks after operation in each group

$(\bar{x} \pm s)$

\begin{tabular}{|c|c|c|c|c|c|c|c|c|c|c|}
\hline \multirow{2}{*}{ 组 别 } & \multicolumn{2}{|c|}{ 白细胞数 $\left(\times 10^{9} / \mathrm{L}\right)$} & \multicolumn{2}{|c|}{ 红细胞数 $\left(\times 10^{12} / \mathrm{L}\right)$} & \multicolumn{2}{|c|}{ 血红蛋白量 $(\mathrm{g} / \mathrm{L})$} & \multicolumn{2}{|c|}{ 红细胞容积 $(\%)$} & \multicolumn{2}{|c|}{ 平均红细胞容积 (fL) } \\
\hline & 第1周 & 第4周 & 第1周 & 第4周 & 第1周 & 第4周 & 第1周 & 第4周 & 第1周 & 第4周 \\
\hline 无菌纱布组 & $9.0 \pm 3.3$ & $10.0 \pm 3.3$ & $5.6 \pm 0.5$ & $7.3 \pm 0.6$ & $115 \pm 8$ & $136 \pm 10$ & $37.2 \pm 2.6$ & $42.8 \pm 2.5$ & $66.4 \pm 5.3$ & $59.1 \pm 3.5$ \\
\hline 速即纱组 & $10.0 \pm 3.3$ & $9.5 \pm 3.6$ & $5.6 \pm 0.3$ & $6.6 \pm 1.1$ & $112 \pm 6$ & $124 \pm 21$ & $36.5 \pm 2.2$ & $38.9 \pm 6.1$ & $65.6 \pm 5.2$ & $58.6 \pm 2.5$ \\
\hline 纤丝组 & $10.2 \pm 5.7$ & $10.0 \pm 2.5$ & $5.6 \pm 0.4$ & $6.4 \pm 1.7$ & $111 \pm 7$ & $118 \pm 31$ & $36.0 \pm 2.0$ & $37.3 \pm 8.7$ & $64.1 \pm 3.1$ & $59.5 \pm 4.5$ \\
\hline \multirow[t]{2}{*}{ 组 别 } & \multicolumn{2}{|c|}{$\begin{array}{c}\text { 平均血红蛋白量 } \\
(\mathrm{pg})\end{array}$} & \multicolumn{2}{|c|}{$\begin{array}{c}\text { 平均血红蛋白浓度 } \\
(\mathrm{g} / \mathrm{L})\end{array}$} & \multicolumn{2}{|c|}{$\begin{array}{l}\text { 血小板数 } \\
\left(\times 10^{9} / \mathrm{L}\right)\end{array}$} & \multicolumn{2}{|c|}{$\begin{array}{c}\text { 活化部分凝血活酶 } \\
\text { 时间 }(\mathrm{s})\end{array}$} & \multicolumn{2}{|c|}{$\begin{array}{l}\text { 凝血酶原 } \\
\text { 时间 }(\mathrm{s})\end{array}$} \\
\hline & 第1周 & 第4周 & 第1周 & 第4周 & 第1周 & 第4周 & 第1周 & 第4周 & 第1周 & 第4周 \\
\hline 无菌纱布组 & $20.5 \pm 1.5$ & $18.8 \pm 0.7$ & $308 \pm 5$ & $318 \pm 10$ & $1487 \pm 165$ & $1221 \pm 184$ & $11.7 \pm 1.4$ & $11.3 \pm 2.5$ & $8.7 \pm 0.4$ & $8.9 \pm 0.9$ \\
\hline 速即纱组 & $20.2 \pm 1.3$ & $18.7 \pm 0.8$ & $309 \pm 11$ & $319 \pm 5$ & $1497 \pm 260$ & $1249 \pm 283$ & $11.8 \pm 1.4$ & $10.8 \pm 2.0$ & $8.7 \pm 0.4$ & $8.5 \pm 0.7$ \\
\hline 纤丝组 & $19.7 \pm 0.6$ & $18.6 \pm 1.1$ & $308 \pm 7$ & $313 \pm 12$ & $1398 \pm 410$ & $1233 \pm 153$ & $13.0 \pm 2.1$ & $12.8 \pm 1.6$ & $8.8 \pm 0.5$ & $8.8 \pm 0.5$ \\
\hline
\end{tabular}

无菌纱布组、速即纱组和纤丝组术后第 1 周统计例数分别为 $10 、 9$ 和 9 只, 术后第 4 周统计例数分别为 $10 、 8$ 和 7 只.

利影响,提示纤丝用于大鼠体内是安全的。

综上,本研究对自主研发的一种氧化再生纤 维素纤丝材料的分子结构及性质进行表征,证实 其具有明显的氧化纤维素分子结构; 与原料相比, 氧化后材料的酸碱度值和相对分子质量显著减 少; 在接触血液后体积膨胀, 可吸收自身质量数十 倍的血液; 细胞实验证实了其具有较好的细胞相 容性; 动物实验显示, 纤丝可实现 $2 \min$ 内快速止 血; 纤丝在植人体内第 1 周即可降解,无组织反应, 可被机体完全吸收,提示具有较高的生物安全性 和止血有效性。因此,该纤丝性能优异,具有较高 的临床应用潜力。

利益冲突 作者王玲爽、童健星、赵哲哲系杭州协合医 疗用品有限公司员工,其他作者声明不存在利益冲突

\section{参考文献}

[1] 张世庆, 赵 熊, 鲁景艳, 等. 可吸收止血材料研究现 状与相关技术要求 $[\mathrm{J}]$. 军事医学, 2012, 36(11): 862-866. ZHANG Shiqing, ZHAO Xiong, LU Jingyan, et al. Review on absorbable hemostatic material and its technical requirements $[\mathrm{J}]$. Military Medical Sciences, 2012, 36(11): 862-866. (in Chinese)

[2] 张 爽, 徐庆华, 童 琳, 等. 可吸收止血材料的研究 现状与应用 $[J]$.中国组织工程研究, 2021，25(10): 1628-1634.

ZHANG Shuang, XU Qinghua, TONG Lin, et al. Current status and application of absorbable hemostatic materials $[\mathrm{J}]$. Chinese Journal of Tissue Engineering Research, 2021, 25(10): 1628-1634. (in Chinese)

[3] HUTCHINSON R W, GEORGE K , JOHNS D, et al.
Hemostatic efficacy and tissue reaction of oxidized regenerated cellulose hemostats $[\mathrm{J}]$. Cellulose, 2013, 20(1): 537-545.

４４中华人民共和国国家质量监督检验检疫总局，中国 国家标准化管理委员会. GB/T 16886.5-2017 医疗器 械生物学评价第 5 部分: 体外细胞毒性试验 $[\mathrm{S}]$. 北京: 中国标准出版社, 2017.

State General Administration of the People's Republic of China for Quality Supervision and Inspection and Quarantine, Standardization administration of China. GB/T 16886.5-2017 Biological evaluation of medical devices part 5: in vitro cytotoxicity test[S]. Beijing: China Standard Press, 2017. (in Chinese)

[5] 董延年, 尹纪业, 陈 长. SD 大鼠脏器重量及脏器系数 正常参考值的确立与应用 $[J]$. 军事医学. 2012, 36(5): 351-353.

DONG Yannian, YIN Jiye, CHEN Chang. Establishment and application of the normal reference values of organ masses and organ/body coefficients in SD rats $[\mathrm{J}]$. Military Medical Science, 2012, 36(5): 351-353. (in Chinese)

[6] MERKEL K, RYDAROWSKI H. FTIR Study and mechanical properties of cellulose fiber-reinforced thermoplastic composites $[\mathrm{J}]$. J Biobased Mater Bio, 2012, 6(4): 399-409.

[7] SEZER U A, SAHIN S, ARU B, et al. Cytotoxicity, bactericidal and hemostatic evaluation of oxidized cellulose microparticles: structure and oxidation degree approach $[J]$. Carbohyd Polym, 2019, 219: 87-94

[8] 彭承宏. 肝切除术中肝脏创面保护和再生氧化纤维 素应用 $[\mathrm{J}]$.中国实用外科杂志, 2014, 34(8): 780-782. PENG Chenghong. Application of liver wound protection and regeneration of oxidized cellulose during liver resection $[\mathrm{J}]$. Chinese Journal of Practical Surgery, 2014, 34(8): 780-782. (in Chinese) 
[9] 黄 伟, 徐小慧, 郭利萍. 三维结构和氧化度对 C6 位 氧化再生纤维素止血材料性能影响 $[\mathrm{J}]$. 高分子通报, 2017, (9): 71-78.

HUANG Wei, XU Xiaohui, GUO Liping. The effect of three-dimensional structure and degree of oxidation on the properties of C6 oxidized regenerated cellulose hemostatic material $[\mathrm{J}]$. Polymer Bulletin, 2017, (9): 71-78. (in Chinese)

[10］翟美玉, 彭 茜. 生物可降解高分子材料 $[\mathrm{J}]$. 化学 与粘合, 2008, 30(5): 66-69.

ZHAI Meiyu, PENG Qian. Biodegradable high polymer material $[\mathrm{J}]$. Chemistry and Adhesion, 2008, 30(5): 66-69. (in Chinese)

[11] ASHTON, WILLIAM H. Oxidized cellulose product and method for preparing the same: United States, 3364200[P]. 1968-01-16.

[12] 王 晨. 生物功能材料在止血方面的应用 $[\mathrm{J}]$. 明胶 科学与技术, 2014, 34(4): 168-174.

WANG Chen. Application of biological functional materials on hemostasis $[\mathrm{J}]$. The Science and Technology of Gelatin, 2014, 34(4): 168-174. (in Chinese)

[13] 童正阳. 可吸收生物止血材料研究进展 $[\mathrm{J} / \mathrm{CD}]$. 世
界最新医学信息文摘(电子版), 2017, 17(2): 43-43.

TONG Zhengyang. Research progress of absorbable biological hemostatic materials $[\mathrm{J} / \mathrm{CD}]$. World Latest Medicine Information (Electronic Editiion), 2017, 17(2): 43-43. (in Chinese)

[14] 张惠影, 陈祥锦, 吴坤林, 等. 再生氧化纤维素预防 麦默通微创旋切术后血肿的临床研究 $[\mathrm{J}]$. 海峡药 学, 2012, 24(10): 143-144.

ZHANG Huihao, CHEN Xiangjin, WU Kunlin, et al. The clinical study of regenerated oxidized cellulose to prevent hematoma after mammotome minimally invasive atherectomy $[\mathrm{J}]$. Strait Pharmaceutical Journal, 2012, 24 (10): 143-144. ( in Chinese)

[15］李 楠, 刘 波, 何 达, 等. 氧化再生纤维素和可 吸收明胶海绵在 ACDF术中的止血作用对比 $[\mathrm{J}]$. 山 东医药, 2014, 54(44): 34-36.

LI Nan, LIU Bo, HE Da, et al. Contrastive analysis of oxidated regenerated cellulose and absorbable gelatin sponge on the hemostasis during ACDF procedure[J]. Shandong Medicine, 2014, 54 (44): 34-36. (in Chinese) [本文编辑 沈 敏 余 方]

\section{叶招明教授团队揭示普通型软骨肉瘤的免疫治疗 获益人群}

近日,浙江大学医学院附属第二医院叶招明教授和林秾主任医师团队在《临床肿瘤研究》(Clinical Cancer Research) 发表了题为 “Fresh tissue multi-omics profiling reveals immune classification and suggests immunotherapy candidates for conventional chondrosarcoma” 的研究论文(https://clincancerres. aacrjournals. org/content/early/2021/10/13/1078-0432. CCR21-1893. long),他们发现 “免疫衰竭” 的普通型软骨肉瘤患者临床可通过程序性死亡蛋白-1(PD-1)抗体治疗而获益。

研究人员应用质谱流式分析绘制了普通型软骨肉瘤的免疫细胞图谱,发现普通型软骨肉瘤是一种富含CD68hi CD11c+树突状细胞的肿瘤, 可分为三种免疫亚型, 即高度免疫抑制的 “G-MDSC优势型”、富含树突状细胞和T细胞的 “免疫衰竭型” 和免疫细胞浸润极少的 “免疫沙漠型”。应用全外显子测序、影像学和病理学数据等多组学手段发现， 异柠檬酸脱氢酶(IDH) $1 / 2$ 突变、磁共振T2相的瘤周水肿、病理WHO II + III 级是普通型软骨肉瘤中存在免疫反应的生物学 标志, 同时符合以上三个条件的肿瘤为 “免疫衰竭” 亚型。在临床治疗中发现 “免疫衰竭型” 普通型软骨肉瘤可能是 免疫治疗的获益对象。

在原本缺乏免疫细胞浸润的鼠源肿瘤中敲人突变IDH能增加肿瘤内免疫细胞数量,且使用以PD-1抗体为基础的免 疫治疗能控制肿瘤生长、延长荷瘤鼠存活期。IDH突变的肿瘤微环境CXCL12浓度增加,树突状细胞加快向肿瘤组织归巢 并接触肿瘤新抗原、开始抗原递呈过程,继而分泌CXCL9、CXCL10等 I 类趋化因子促进外周T细胞向肿瘤组织归巢,从而 发挥杀伤肿瘤作用。

李冰皓主治医师、李国奇博士、严晓波副主任医师为论文第一作者。研究得到国家自然科学基金资助。 